\title{
Representing the light field in finite three-dimensional spaces from sparse discrete samples
}

\author{
Alexander A. Mury, ${ }^{1, *}$ Sylvia C. Pont, ${ }^{1}$ and Jan J. Koenderink ${ }^{2}$ \\ ${ }^{1}$ Faculty of Industrial Design Engineering, Delft University of Technology, \\ Landbergstraat 15, 2628CE Delft, The Netherlands \\ ${ }^{2}$ Faculty of Electrical Engineering, Mathematics, and Computer Science, Delft University of Technology, \\ Mekelweg 4, 2828 CD Delft, The Netherlands \\ ${ }^{*}$ Corresponding author: a.muryy@tudelft.nl
}

Received 16 September 2008; revised 19 November 2008; accepted 25 November 2008; posted 11 December 2008 (Doc. ID 101674); published 12 January 2009

\begin{abstract}
We present a method for measurement and reconstruction of light fields in finite spaces. Using a custommade device called a plenopter, we can measure spatially and directionally varying radiance distribution functions from a real-world scene up to their second-order spherical harmonics approximations. Interpolating between measurement points, we can recover this function for arbitrary points of a scene. We visualized the global structure of the light field using light tubes, which gives an intuitive description of the flux propagation throughout three-dimensional scenes and provides information about the quality of light in the scenes. Our second-order reconstructions are sufficient to render convex matte objects and therefore have a direct interest for computer graphics applications. () 2009 Optical Society of America OCIS codes: $120.5240,150.2950$.
\end{abstract}

\section{Introduction}

The radiance distribution throughout the empty space of a three-dimensional (3D) scene is a complicated function and therefore rarely mapped out empirically. However, knowing the light field [1,2] of a real scene (or at least some of its qualitative properties) is important for many applications: an interior designer can predict the appearance of an object placed at some arbitrary point in that scene or an architect can make a decision whether the building satisfies the standards. In computer graphics, the light field is important for rendering purposes; imagine an object moving through a scene, its appearance changes due to illumination variations along its trajectory. The complexity of the light field makes it difficult to measure and analyze it even at a point. Recovering this function for every point of a finite $3 \mathrm{D}$ space is a challenging and tedious (but not impossible) task. However, the complete description is over-

0003-6935/09/030450-08 $\$ 15.00 / 0$

(C) 2009 Optical Society of America kill for most applications. Instead, we limit the description of the structure of the light field to components that are the most qualitatively important for object appearance and that suffice to represent the flux distribution throughout the scene. This simplification allows us to develop a description that gives a clear understanding of the structure of the light fields in 3D scenes.

Quantitative assessment of the visual quality of the luminous environment is typically concerned with measuring illumination incident on surfaces $[3,4]$. The radiance distribution in the empty space of a scene is rarely taken into account. The empirical analysis of the structure of light fields in empty space is usually limited to measuring the space illumination of radiation and the light vector at a point [3-6]. In general, radiant flux propagates from light sources to light-absorbing surfaces (however, in some special cases, which rarely occur in real scenes, flux lines can be closed). Although it does not produce any visual effect in empty space, the distribution of radiance explains the "spatial and form-giving character" of light. Knowing the radiance distribution, one could 
predict the appearance of a hypothetical object placed somewhere in space and calculate irradiance patterns over its surface.

We present a new way of measuring and analyzing the structure of light fields in finite 3D spaces. Our custom-made device, called a plenopter, is capable of measuring local light fields up to the second order in terms of spherical harmonics. This extends the conventional description by a new parameter-the squash tensor (the second-order harmonic [7]). Taking into account that the low-order components of light fields in natural scenes typically vary slowly and rather systematically with location [7], the second-order approximation of the radiance distribution function can be estimated reasonably well for all points of the scene using interpolation between a limited number of observations. In order to describe the global structure of the light field of a scene, we utilize the concept of light tubes, which provides an easy and intuitive understanding of the propagation of radiant flux throughout the scene. Although this concept is not new [1], it has never been used for applications before.

We applied this method to several simple and complicated light fields. We rendered objects in those scenes in order to make a connection between our formal description and object appearance.

\section{Previous Works}

The first theory about the light field was developed by Gershun [1]. In his classical work on photometry, Gershun considered the light field in empty space. The complete description of the local light field at a certain point in space is given by the radiance distribution $R(\vartheta, \varphi)$ (brightness-distribution solid, according to Gershun), which is a spherical function representing the radiance arriving at the point from all directions. Gershun considered the light field as a vector field in $3 \mathrm{D}$ space. The most basic property of the local light field is a scalar parameter, namely, the space illumination $d=\int_{4 \pi} R(\vartheta, \varphi) \mathrm{d} \omega$. The vector characteristic of the light field is called the light vector and is given by $v=\int_{4 \pi} R(\vartheta, \varphi) \mathrm{d} \omega$. The direction of the light vector coincides with the direction of maximum flux transfer, and the magnitude is the flux density in that direction (i.e., the difference in irradiance of the two sides of a hypothetical infinitesimal diaphragm). Knowing the light vector at any point in space, one can describe the structure of flux transfer throughout space by constructing flux lines that are tangential to the light vector. Flux lines are curved lines in $3 \mathrm{D}$, and they typically originate on light sources and end on light-absorbing surfaces. Figure 1 shows the flux lines calculated for an artificial street scene with buildings on both sides under uniform overcast illumination; the walls were assumed to be Lambertian with constant albedo, and the flux lines in the areas that are not exposed to the primary light source are due to interreflections. Such a diagram gives an immediate impression of the flux distribution throughout the scene and also provides

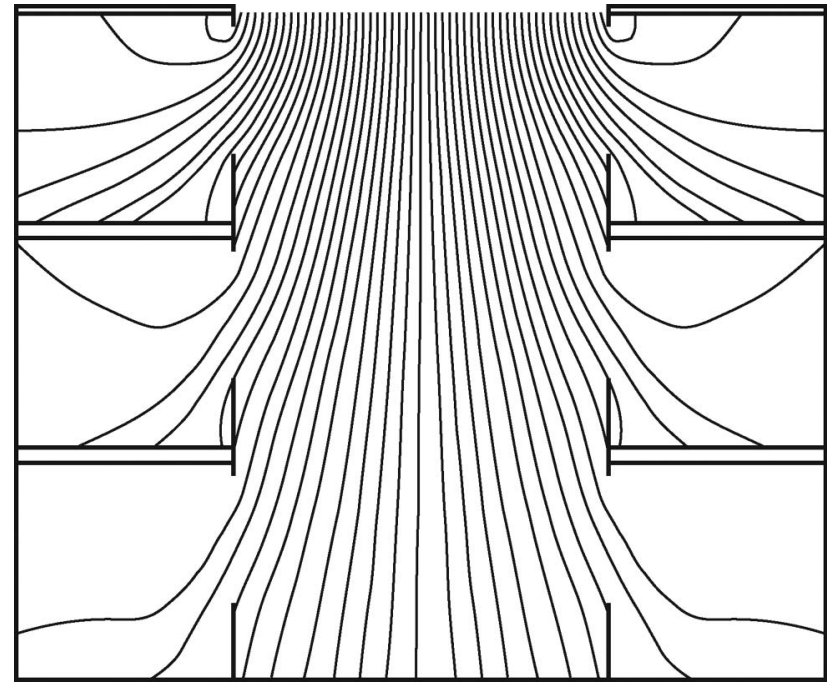

Fig. 1. Example of flux lines-the distribution of the flux lines inside two three-story buildings opposite each other (shown as cross sections) under an overcast sky. The flux lines start at the overcast sky and enter the buildings through the windows. The interreflections are taken into account.

indirect information about the illumination on surfaces-the denser the flux lines, the stronger the illumination. Gershun [1] considered several cases of light fields theoretically, and using calculus, he derived light fields for generic light sources like an infinite luminous stripe and a wall. But he never applied the concepts of light vector and flux lines to describe lighting conditions in real scenes-at his time, such empirical analysis was difficult to realize practically.

In computer science, the light field is known as the plenoptic function [8], which is a function $L(x, y, z, \vartheta, \varphi, \lambda, t)$ of seven variables that indicate position ( $x, y$, and $z$ coordinates); direction $(\vartheta$ and $\varphi$ spherical coordinates); frequency $(\lambda)$; and time $(t)$. A recently developed technique [9] allows us to photographically capture a simplified five-dimensional (5D) plenoptic function $L(x, y, z, \vartheta, \varphi)$ over a certain region of space by recording intensities of all rays passing through the scene. The resulting light field is used in computer graphics for rendering applications [10]. This method exploits the idea that the radiance of a ray is constant along its direction. The disadvantage of this so-called lumigraph method is that the measurement procedure is rather complicated and the postprocessing involves analysis of huge amounts of data. However, up to now, this is the only method of measuring global light fields within finite spaces of real 3D scenes.

A local light field at a point in empty space can also be acquired by means of high dynamic range panoramic imaging [11,12]. Unger et al. [13] suggested the use of an array of mirror balls in order to capture spatially and directionally varying incident light fields over two-dimensional (2D) areas and then extrapolate the data to a volume above the area. These methods are used to model light fields for 
image-based rendering applications in computer graphics.

Practically, detailed descriptions of light fields are needed only to render highly reflective mirrorlike materials. However, for most materials, highfrequency lighting is not necessary, because it will be blurred by diffuse scattering of the illuminated object (a smooth bidirectional reflectance distribution function works as a low-pass filter). This effect has been shown empirically $[14,15]$ and has been derived theoretically - the second-order spherical harmonics approximation of local light fields suffices to render convex Lambertian objects $[16,17]$. Moreover, such low-order approximations allow us to derive the most salient qualitative features of the light field, such as the overall levels and directions of the illumination. The zeroth- and first-order components have direct physical meanings, namely, the space illumination and the light vector (see Fig. 2). The second-order contribution is more complicated and can correspond to different shapes of brightness-distribution solids from a "light clamp" to a "light ring." It is referred to as a "squash tensor" [7]. In natural scenes, the low-order contributions to light fields show very systematic and smooth behavior varying very slowly from one point of the scene to another $[7,18]$.

The field of knowledge that deals with the formal quantifiable assessment of the visual quality of the environment is lighting engineering. A large number of measurable parameters are being used to judge the suitability of the illumination for visual tasks the observer may encounter, e.g., illuminance values, cylindrical illuminance, and daylight factor $[3,4]$. However, lighting engineers are mostly concerned with the illumination incident on surfaces and rarely interested in the radiance distribution in empty space, which is an important factor in the visual assessment of the environment [19]. To the best of our knowledge, there is only one commercially available device that can be used for measurements of the light vector and space illumination [6].

Light fields in empty space are also important in architecture and interior design. In this field, a more artistic approach is used to estimate "spatial and form-giving effects" of lighting. Sample objects are frequently used, and the quality of light is judged from the effects that light field cast upon those

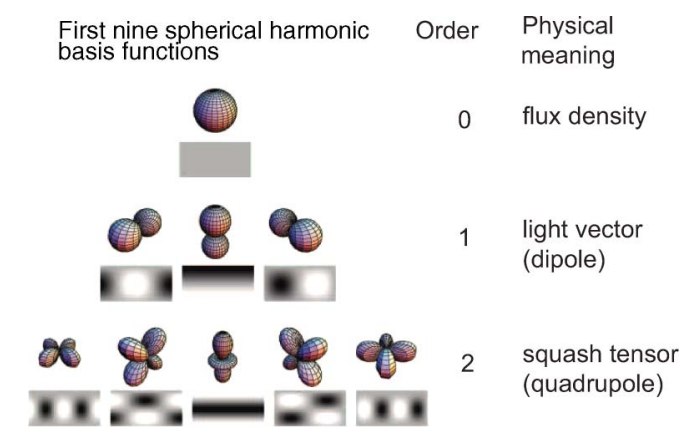

Fig. 2. (Color online) First nine spherical harmonics basis functions. objects. Cuttle [4] suggested the use of three kinds of sample object-a matte sphere, a black shiny sphere, and a disk with a peg in order to reveal shading, highlights, and shadow patterns.

Another way of analyzing illumination is via computer modeling. Physically based rendering systems [20] provide tools to calculate and analyze any parameter of the light field. For instance, one can calculate the light vectors at points of a regular grid. Visualization of those vectors provides a quantitative description of the flux transfer throughout the scene [21]; however, this method has certain drawbacks. Light vectors at discrete points do not reveal a global description of the flux transport. Besides, an image consisting of projections of $3 \mathrm{D}$ vectors on a $2 \mathrm{D}$ image plain is visually ambiguous, and therefore it is difficult to grasp correct orientations and magnitudes of the light vectors from such a representation unless all the vectors are coplanar and lie in the image plane. We suggest another method of depicting flux through a $3 \mathrm{D}$ scene by visualization of light tubes. This not only gives continuous (along a flux line) information about the light vector, but also makes it easier to see variation of its orientation and strength in $3 \mathrm{D}$.

The irradiance volume [22] is a method of recovering the irradiance distribution solid for any point within a virtual scene by means of interpolation. It exploits the idea that the irradiance distribution function (a spherical function representing the irradiance on a hypothetical plane for all possible orientations of that plane) is smooth and therefore easy to interpolate. We use a similar approach and apply interpolation to recover second-order approximations of the radiance distribution functions at all points within a certain volume.

\section{Empirical Studies}

The goal of our empirical studies is to reconstruct the light field up to the second-order approximation at any point inside a $3 \mathrm{D}$ space of a scene and to provide insight into the global structure of the light field. We considered several light fields and provide a detailed description of the second-order approximations of those light fields. We use a custom-made device called a plenopter (Fig. 3). The plenopter contains 12 high dynamic range sensors in a regular dodecahedron configuration. Each sensor has a large field of view of $74^{\circ}$, such that, altogether they cover the entire sphere, capturing light that is coming to its center point from all directions. Each sensor provides one value representing an average intensity over the field of view. The sensors are radiometrically calibrated. The plenopter is capable of measuring the structure of the local light field up to the second order in terms of spherical harmonics. From a single plenopter sample (12 values), we can calculate nine spherical harmonic coefficients representing the zeroth-, first-, and second-order contributions at the measurement point. 


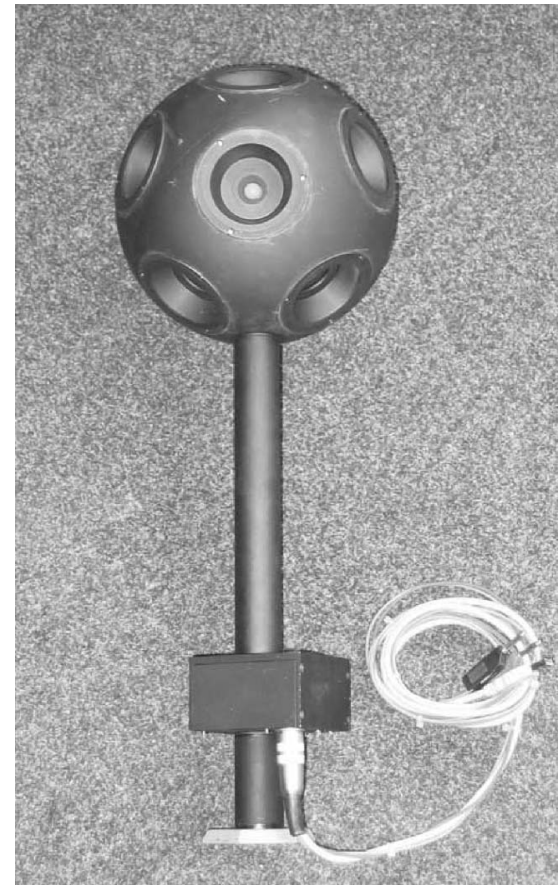

Fig. 3. Plenopter: a light-measuring device capable of capturing local light fields up to the second order. Twelve photocells with wide apertures of $74^{\circ}$ cover the entire sphere capturing light from all directions.

The empirical studies were conveyed in the Light Lab (Philips Research), which is a typical empty office room $4 \mathrm{~m} \times 6 \mathrm{~m} \times 3 \mathrm{~m}$, equipped with a large number of different kinds of light sources that could be controlled remotely. The equipment of the Light Lab allowed us to generate 24 qualitatively different light fields. We applied our analyses to all of them but here show only four that represent the most extreme cases. Schematic outlines of the scenes and photographs of a mirror ball taken in the middle of the room are shown in Fig. 4. The light sources of Scene A were three square area light sources on the ceiling close to the left wall [Fig. 4(a)]. In Scene $\mathrm{B}$, there was one large diffuse circular light source in the middle of the ceiling [Fig. 4(b)]. The illumination of Scene C consisted of four spotlight sources positioned at the corners of the room and directed downward [Fig. 4(c), notice large bright areas on the walls and floor that are directly illuminated). In Scene D, we used three area light sources of the same kind as in the first scene but in a triangular instead of a linear configuration [Fig. 4(d)].

The measurement procedure was as follows. We took 45 plenopter measurements over a regular grid at three height levels $(100,155$, and $210 \mathrm{~cm})$. At each level there were 15 points (array 3 by 5 points) $1 \mathrm{~m}$ apart. For Scenes A and D, the middle level was again measured a few months later on a finer grid in order to check the repeatability of the measurements and to find out how good the interpolation is by comparing interpolated data with real measurements at extra points. For any point inside the space enveloped by the measurement points, the light field

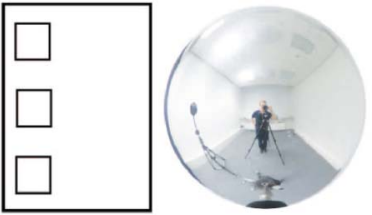

(a)

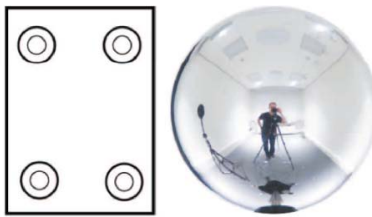

(c)
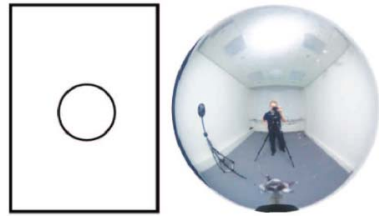

(b)
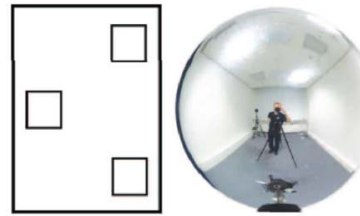

(d)

Fig. 4. (Color online) Schematic description of the light sources (view from above) and panoramic photographs of the Light Lab for different lighting installations. (a) Three diffuse area light sources on the ceiling along the left wall. (b) Large circular diffuse area light source in the middle of the ceiling. (c) Four small spotlights (close to collimated) at the corners on the ceiling directed straight downward. (d) Three diffuse area light sources on the ceiling in a triangular configuration.

was calculated by means of linear interpolation of the coefficients between the neighboring measurement points.

\section{Results}

Figure 5 shows contour plots representing the magnitudes of the first three harmonics (space illumination, light vector, and squash tensor) over the plain at a height of $155 \mathrm{~cm}$ (middle level) for all four scenes. The white circles indicate the 15 measurement points taken on this level. Notice that the values of space illumination nicely correspond to the layouts of the scenes (Fig. 4) - the closer to the light source, the stronger it gets. Typically, the distributions of the magnitudes for the vectors are similar to those of the
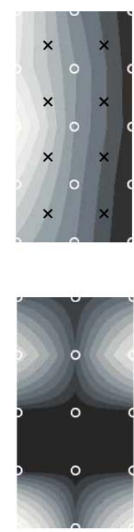

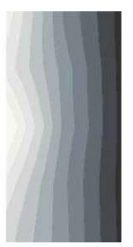

(a)

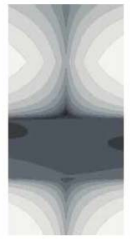

(c)
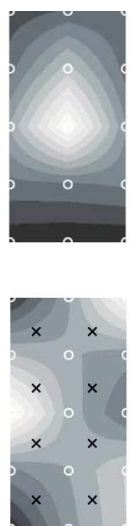

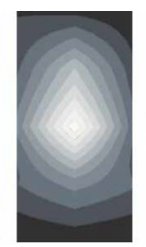

(b)

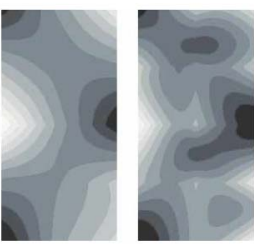

(d)

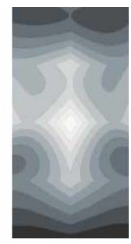

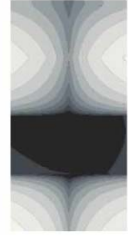
Fig. 5. Logarithmic contour plots representing the magnitudes of
space illumination (left), light vector (middle), and squash tensor (right) over the middle level $(155 \mathrm{~cm}$ ) for scenes depicted in Fig. 4. The magnitudes were scaled individually such that the maximum range of gray values is used. White crosses indicate the measurement points used for interpolation; black crosses indicate the extra measurements taken in Scenes A and D. 
space illumination (Fig. 5). However, in general, that does not always have to be true.

The light vector may vanish at points where the local light fields are symmetrical, for instance, in the middle between two identical light sources. The structure of the squash tensor and its behavior are more difficult to interpret than the previous two -in fact, the higher the order, the more complicated it gets. However, there are two canonical cases of the squash tensor that could be named "light clamp" and "light ring" that have simple geometric interpretations and can help to understand the behavior of the squash tensor. For instance, in the middle of Scenes A and D, the squash tensor has local maxima, which, as we can judge from the geometric layouts of the scenes, are due to a strong "light clamp" at those points.

In order to test how good the interpolation is, we took eight extra measurements in between the grid points for Scenes A and D. The locations of extra measurements are indicated by black crosses in Figs. 5(a) and 5(d). Figure 6 shows the comparison of the nine spherical harmonic coefficients calculated from real measurements (light gray bars) at those extra points against coefficients calculated by using interpolation (dark gray bars) on the basis of the 45 original samples. As can be seen, the measured and interpolated coefficients correspond rather well even for Scene D, which is structurally complicated; mean correlations between the vectors of interpolated and measured spherical harmonic coefficients were 0.89 for Scene A and 0.92 for Scene D.
The errors can be partially explained by the large step size of the grid-we used $3 \times 5 \times 3$ grids in steps of $1 \mathrm{~m}$. Obviously, complicated light fields that consist of rather collimated light sources with beam widths of tens of centimeters (like Scene C) would require a finer grid. There are also errors due to the misplacement of the device; the accuracy of the plenopter's location was $\pm 5 \mathrm{~cm}$ in $3 \mathrm{D}$. At special points of the light field, for instance, on the light-dark edge in the case of collimated light, such misplacement of the device could lead to significant errors.

It is rather difficult to understand the shapes of the light fields purely from the coefficients, and therefore Fig. 7, showing panoramic pictures $\left(360^{\circ} \times 180^{\circ}\right)$, which represent the spherical radiance distribution functions up to the second-order approximations, corresponding to the coefficients shown in Figs. 6 and 7, demonstrates that the interpolated spherical functions capture the qualitative structure of local light fields very well (notice that bright and dark patches on contour plots corresponding to measured and interpolated coefficients coincide). So although the errors in the individual spherical harmonic coefficients are sometimes large (Fig. 6), they hardly ever lead to serious errors in the global structure of the second-order approximation of the spherical function, which is the summation of all nine spherical harmonics basis functions weighted by the coefficients.

Figure 8 shows the light tubes for all four scenes. The light tubes are constructed in such a way that they are always tangential to the light vector, and
Scene A
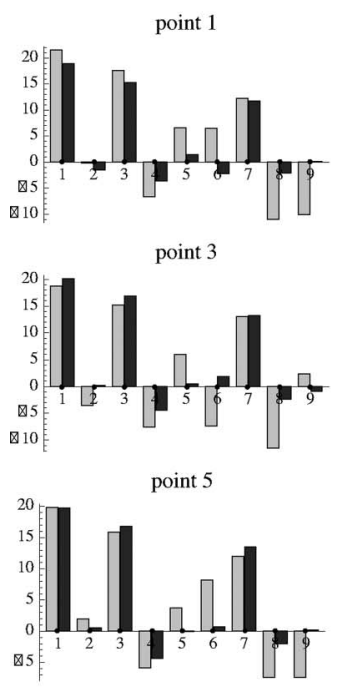

point 7

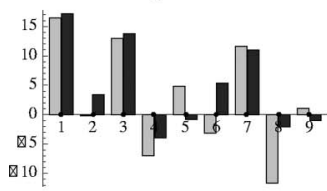

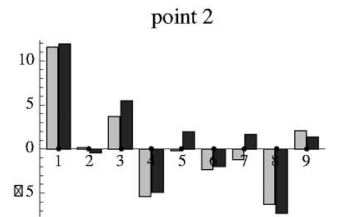

point 4

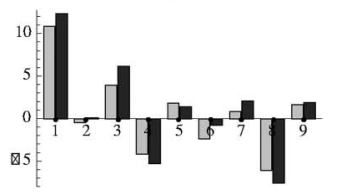

point 6

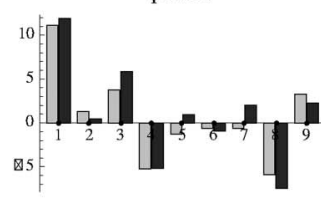

point 8

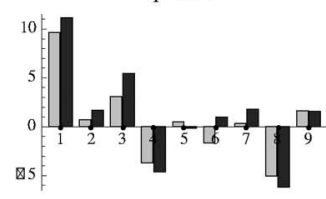

Scene D

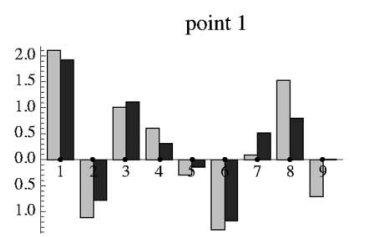

point 3

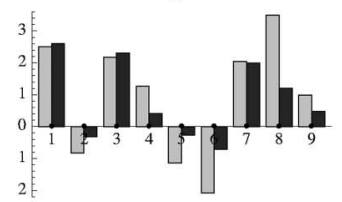

point 5

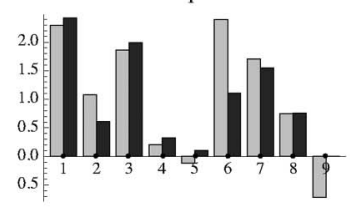

point 7

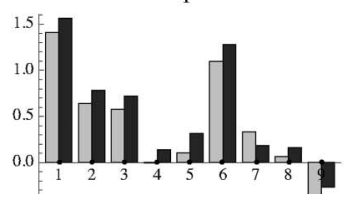

point 2

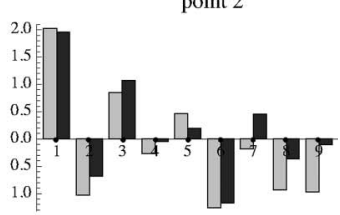

point 4

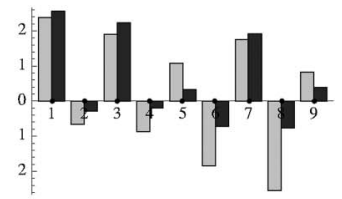

point 6

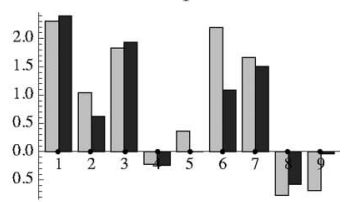

point 8

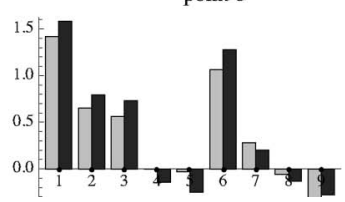

Fig. 6. Spherical harmonics coefficients calculated in Scenes A (left) and D (right) at extra points indicated by crosses on Fig. $\underline{5}$. Light gray bars represent spherical harmonics calculated from real measurements and dark gray from interpolated values. 


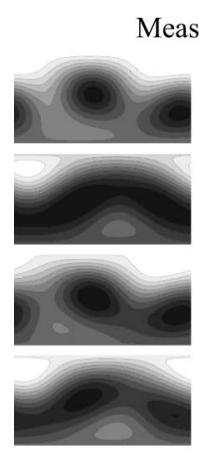

\section{ured}
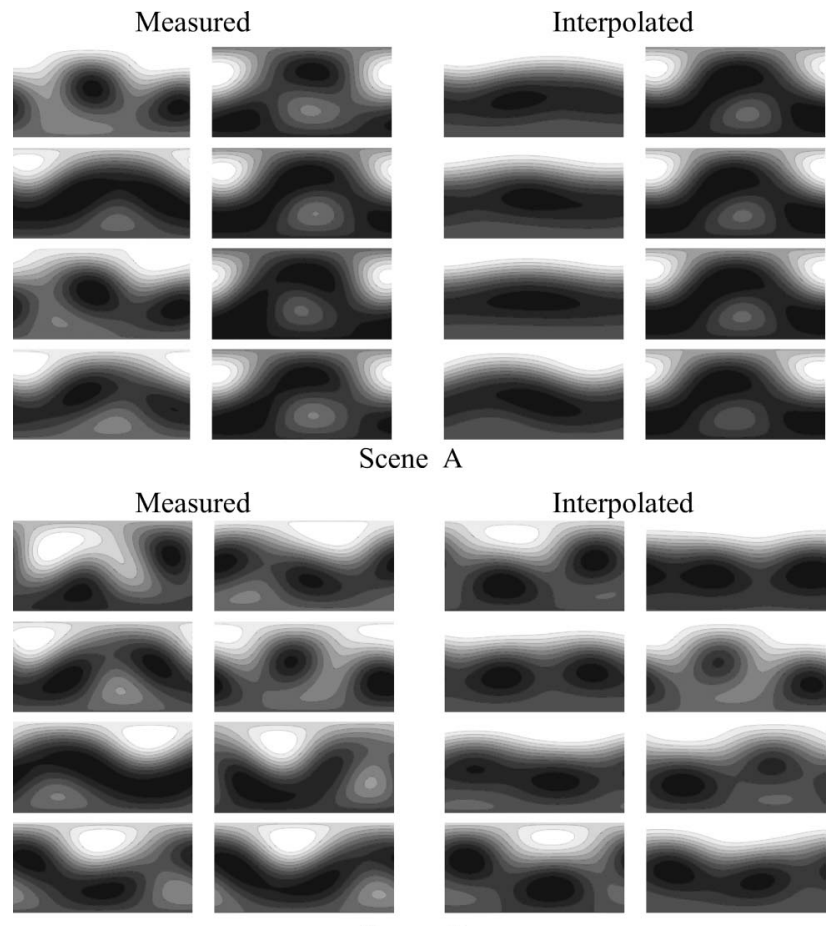

Scene D

Fig. 7. Panoramic $180^{\circ} \times 360^{\circ}$ plots representing second-order approximations of the light fields corresponding to the coefficients in Fig. $\underline{6}$ (left, real measurements; right, interpolated) for Scenes A and $\mathrm{D}$.

the radius of the tubes is inversely proportional to the magnitude of the light vector $r=1 / \sqrt{\pi\|v\|}$, meaning that the flux through orthogonal cross sections of the tube is constant. It is useful to think of the light flux through the light tube as incompressible fluid flow through a tube; the amount of flux through an element of the tube is constant, independent of the width of that element, however, the speed varies inversely proportional to the radius of the tube. In this case, the magnitude of the light vector is analogous to the flow velocity. In Fig. 8 , drawn squares and circles on the ceiling indicate the light sources (notice that the light tubes correspond very well to the schematics in Fig. 4). The overall qualitative descriptions of the flux transfer by light tubes are captured correctly for all scenes, as we can judge from the geometric layouts of the scenes.

In order to capture all flow features, a seed placement strategy for the flux lines should be chosen wisely [23]. The resulting image of the light tubes depends on this choice. For instance, if for Scene $\mathrm{C}$ we would have chosen starting points on the left or right walls, then perhaps none of the constructed light tubes would have passed through the middle of the room (notice that there are almost no tubes ending on the walls), and therefore the flow pattern in that area would be missing in such visualization. This problem usually occurs around critical points of the field. We have chosen a $5 \times 9$ array of points on the ceiling for all the scenes, because the flux lines

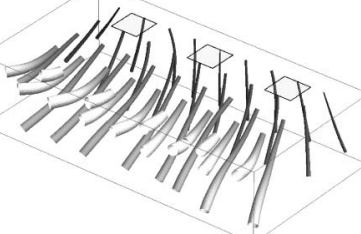

(a)

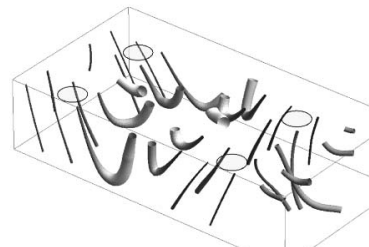

(c)

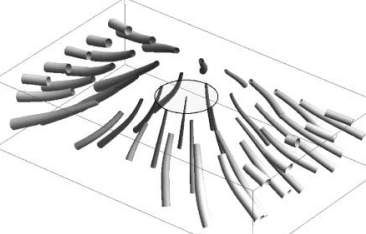

(b)

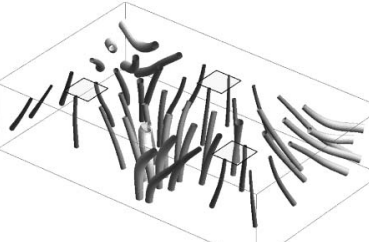

(d)
Fig. 8. Light tubes for the scenes schematically depicted in Fig. 4 . The light sources are indicated by squares and circles. The tubes describe radiant flux transfer. Flux through any section of any light tube within a scene is constant. The light vectors are tangential to the tubes, and their magnitudes are counter proportional to the square areas of tubes sections.

typically originate at the light sources (on the ceiling in our case).

Notice that in Scene $\mathrm{C}$ some light tubes are curved as parabolas starting at the ceiling and ending on the ceiling, meaning that the light vector changed its direction almost $180^{\circ}$. In the middle of that scene, the direction of flux transfer is upward due to strong reflections from the ground. The flux in the upper middle part of Scene $\mathrm{C}$ is very low (light tubes are thick), because the light field there is mostly due to secondary light sources, and the strong narrow beams of the primary light sources do not reach that part of the room.

The light tubes provide an intuitive visualization of the light field behavior over the scene and of how the flux propagates through the scene; however, they do not provide direct information about the space illumination and the squash tensor. Figure 9 shows how the full second-order approximation of the light field varies along a tube. We have chosen one light tube from Scene C [Fig. 9(a)] and considered three points along that tube. Figure 9(b) shows the corresponding radiance distribution functions up to the second-order approximations and images of a Lambertian object (the Stanford Bunny) rendered using those second-order light fields. From the appearance of the rendered objects, we can clearly see how the primary direction of light changes along the tube. In the lowest part of the tube, there is a clear "light clamp" structure (notice that the light field at that point has two maximums that are opposite each other). The clamp is also visually apparent from the object's appearance.

\section{Discussion and Conclusions}

We present a new technique for measuring light fields and recovering the second-order approximation over a finite region of $3 \mathrm{D}$ space. Our 


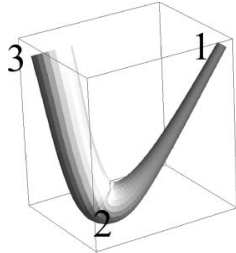

(a)
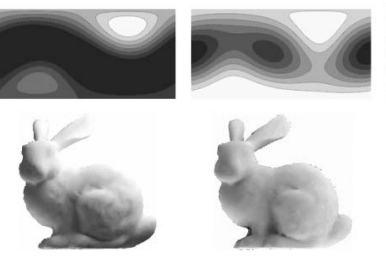

(b)

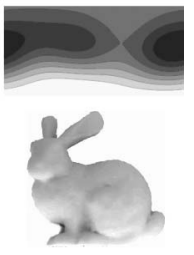

Fig. 9. Object's appearance along a light tube. (a) One of the light tubes selected from Scene C. (b) Pictures of Lambertian 3D object (Stanford Bunny) rendered using light fields at points indicated on the tube. The contour plots represent $180^{\circ} \times 360^{\circ}$ panoramic images of the second-order light fields at those points.

second-order spherical harmonics approximations provide quantitative mathematical descriptions of important structural features of the light field, which allows us to approach the "spatial and form-giving effects" of lighting formally. The major contribution is the possibility to estimate the light field not only locally at a point of space, but globally over the entire space.

We can think of many applications for our technique. Since the light tubes describe the flux propagation throughout a $3 \mathrm{D}$ scene, it is a useful addition to the tools of lighting engineers. Light tubes provide a qualitative description of the global structure of light fields and also contain quantitative information about the strength of the light vectors. Although light tubes do not give direct information about the space illumination, some information about the space illumination can be retrieved from the light tubes indirectly. Typically there is a correlation between the density of the flux lines and the local maxima of space illumination (this does not hold for singular points, for instance, where the light vector vanishes). In addition to measuring these well-known structural features of the light field, our method also allows us to estimate the squash tensor. To the best of our knowledge, the available light-measuring devices are capable of measuring only light vector and space illumination locally, therefore the possibility of recovering the higher-order structural component (squash tensor) takes us one step farther in understanding the quality of light.

Architects and interior designers may also find this technique useful for evaluating the quality of light in a scene globally. Our method allows us to find special points in the light field, for instance, where the light vector vanishes and the structure of the local light field is dominated by a squash tensor. This is a formal scientific approach to express the quality of light in the scene numerically, whereas the "flow of light" $[\underline{4}, 24]$, which is based on object appearance, provides only a qualitative description. Besides, the second-order spherical harmonics description can be used as lighting input for rendering systems.

In computer graphics, our technique could be used as a cheap and quick alternative to the Lumigraph. The angular resolution of the plenopter is low; however, our method provides the global structure of the
$5 \mathrm{D}$ light field in a finite space on the basis of just tens of measurements of 12 numbers, which can be done in less than an hour, without the need for much memory space and heavy computations. Moreover, our methods provide direct intuitive insights into the global structure of light fields-which is not the case for high-resolution methods such as the lumigraph.

Our measurements showed that even the structure of the first-order component of the light field, which is given by the light tubes, can be rather complicated. Notice that the light vector changes its direction almost $180^{\circ}$ [Fig. 8(c)] starting at the ceiling and ending up on the ceiling as well. Theoretically the flux lines can even be closed. In future research, we will investigate possible topological structures of the light field and analyze its singular points. Furthermore, we will study the intriguing relations between human perception of the luminous environment and structural features of the light fields.

This work was supported by the Netherlands Organization for Scientific Research (NWO). We thank H. Kolijn for assisting us with technicalities. We also thank M. Reisinger and I. Vogels of the Visual Experiences Group at Philips Research, Eindhoven, The Netherlands, for collaboration and providing their outstanding facilities.

\section{References}

1. A. Gershun, "The light field," Moscow (1936), translated by P. Moon and G. Tinoshenko in J. Math. Phys. 18, 51-151 (1939).

2. P. Moon and D. E. Spencer, The Photic Field (MIT, 1981).

3. P. R. Boyce, Human Factors in Lighting (Macmillan, 1981).

4. C. Cuttle, Lighting by Design (Architectural, 2003).

5. C. Cuttle, "Cubic illumination," Light. Res. Technol. 29, 1-14 (1997).

6. http://www.megatron.co.uk/cim/index.html.

7. A. A. Mury, S. C. Pont, and J. J. Koenderink, "Light field constancy within natural scenes," Appl. Opt. 46, 7308-7316 (2007).

8. E. H. Adelson and J. Bergen, "The plenoptic function and the elements of early vision," in Computational Models of Visual Processing, M. Landy and J. Movshon, eds. (MIT, 1991), pp. 3-20.

9. S. J. Gortler, R. Grzeszczuk, R. Szeliski, and M. F. Cohen, "The lumigraph," in Computer Graphics, Proc. SIGGRAPH 96 (1996), pp. 43-54.

10. M. Levoy and P. Hanrahan, "Light field rendering," in Proc. SIGGRAPH 96 (1996), pp. 31-42.

11. http://www.debevec.org/Probes/.

12. P. Debevec, "Rendering synthetic objects into real scenes: bridging traditional and image-based graphics with global illumination and high dynamic range photography," in Proc. SIGGRAPH 98 (1998), pp. 189-198.

13. J. Unger, A. Wenger, T. Hawkins, A. Gardner, and P. Debevec, "Capturing and rendering with incident light fields," in Proc. of the 14th Eurographics Workshop on Rendering (2003).

14. R. Epstein, P. Hallinan, and A. Yuille, "5 plus or minus 2 eigenimages suffice: an empirical investigation of lowdimensional lighting models," in Proc. IEEE Workshop Physics-Based Modeling in Computer Vision (1995), pp. 108-116.

15. P. W. Hallinan, "A low-dimensional representation of human faces for arbitrary lighting conditions," in Proc. IEEE Confer- 
ence on Computer Vision and Pattern Recognition (1994), pp. 995-999.

16. R. Ramamoorthi and P. Hanrahan, "On the relationship between radiance and irradiance: determining the illumination from images of a convex Lambertian object," J. Opt. Soc. Am. A 18, 2448-2459 (2001).

17. R. Basri and D. Jacobs, "Lambertian reflectance and linear subspaces," Proc. 8th IEEE Int. Conf. Computer Vision (2001), pp. 383-390.

18. A. A. Mury, S. C. Pont, and J. J. Koenderink, "Spatial properties of light fields in natural scenes," in Proc. APGV 2007, ACM SIGGRAPH, S. N. Spencer, ed. (2007), p. 140.

19. J. J. Koenderink, S. C. Pont, A. J. van Doorn, A. M. L. Kappers, and J. T. Todd, "The visual light field," Perception 36, 15951610 (2007).
20. G. W. Larson and R. A. Shakespeare, Rendering with Radiance: the Art and Science of Lighting Visualization (Morgan Kaufmann, 1997).

21. A. Jacobs, MSc in Energy, Architecture and Sustainability: European Masters in the Integration of Renewable Energies into Buildings, RADIANCE Course (Advanced) (London Metropolitan University, 2004).

22. G. Greger, P. Shirley, P. Hubbard, and D. Greenberg, "The irradiance volume," IEEE Comput. Graph. Appl. 18, 32-43 (1998).

23. V. Verma, D. Kao, and A. Pang, "A flow-guided streamlines seeding strategy," Proc. IEEE Visualization (2000), pp. $163-170$.

24. C. Cuttle, "Lighting patterns and the flow of light," Light. Res. Technol. 3, 171-189 (1971). 